\title{
Evaluation of morbidity, mortality and outcome following cervical spine injuries in elderly patients
}

\author{
S. A. Malik $\cdot$ M. Murphy $\cdot$ P. Connolly $\cdot$ \\ J. O'Byrne
}

Received: 23 January 2006/Revised: 30 July 2007/ Accepted: 22 December 2007/Published online: 15 January 2008

(c) Springer-Verlag 2008

\begin{abstract}
We analysed the morbidity, mortality and outcome of cervical spine injuries in patients over the age of 65 years. This study was a retrospective review of 107 elderly patients admitted to our tertiary referral spinal injuries unit with cervical spine injuries between 1994 and 2002. The data was acquired by analysis of the national spinal unit database, hospital inpatient enquiry system, chart and radiographic review. Mean age was 74 years (range 66-93 years). The male to female ratio was 2.1:1 $(\mathrm{M}=72, \mathrm{~F}=35)$. The mean follow-up was 4.4 years $(1-$ 9 years) and mean in-hospital stay was 10 days (290 days). The mechanism of injury was a fall in 75 and road traffic accident in the remaining 32 patients. The level involved was atlanto-axial in 44 cases, sub-axial in 52 cases and the remaining 11 had no bony injury. Multilevel involvement occurred in 48 patients. C2 dominated the single level injury and most of them were type II odontoid fractures. Four patients had complete neurology, 27 had incomplete neurology, and the remaining 76 had no neurological deficit. Treatment included cervical orthosis in 67 cases, halo immobilization in 25 , posterior stabilization in 12 patients and anterior cervical fusion in three patients. The overall complication rate was $18.6 \%$ with an associated in-hospital mortality of $11.2 \%$. The complications included loss of reduction due to halo and Minerva loosening, non-union and delayed union among conservatively treated patients, pin site and wound infection, gastrointestinal bleeding and complication due to associated injuries. Among the $28.9 \%$ patients with neurological involvement, $37.7 \%$ had significant neurological recovery. Outcome was
\end{abstract}

S. A. Malik $(\bowtie) \cdot$ M. Murphy · P. Connolly · J. O’Byrne National Spinal Injury Unit, Mater Misericordiae Hospital, 59 Eccles Street, Dublin 2, Ireland

e-mail: samalik64@hotmail.com assessed using a cervical spine outcome questionnaire from Johns Hopkins School of Medicine. Sixty-seven patients (70\%) completed the form, 20 patients (19\%) were deceased at review and 8 patients $(7 \%)$ were uncontactable. Functional disability was more marked in the patients with neurologically deficit at time of injury. Outcome of the injury was related to increasing age, co-morbidity and the severity of neurological deficit. Injuries of the cervical spine are not infrequent occurrence in the elderly and occur with relatively minor trauma. Neck pain in the elderly patients should be thoroughly evaluated to exclude C2 injuries. Most patients can be managed in an orthosis but unstable injuries require rigid external immobilization or surgical stabilization.

Keywords Elderly · Cervical spine injury · External immobilization · Outcome

\section{Introduction}

Cervical spine injuries are frequent in the elderly population and in the majority of cases occur following minor trauma. This fact has been attributed to the propensity for the elderly to sustain simple falls, a greater risk for motor vehicle accidents and the biochemical attrition associated with senile osteopenia, most commonly due to primary osteoporosis. The prevalence of cervical spine injuries in the elderly is expected to progressively increase in Europe and North America, as population demographics change. In elderly patients the injury patterns are different from that among younger patients. This is due to the difference in bone density, injury mechanism and presence of degenerative changes. Injuries due to external causes can result in injury deaths and cases of permanent disability. This is 
especially true for neck injuries, with the potential to cause spinal cord trauma. At present, data from different parts of the world point at decreasing neck injury incidence trends for age groups under 65 years, while the incidence is constant or increasing in the elderly. Cervical spine trauma can be devastating when a patient sustains neurological sequelae. It is estimated that $2-3 \%$ of all trauma patients sustain cervical spine injuries and, of those, between 3 and $25 \%$ suffer extension of those injuries from delays in diagnosis or unwarranted manipulation in the emergency department. The management of cervical spine injuries in elderly patients may be complicated by a number of factors, including pre-existing medical conditions such as cardiopulmonary compromise, diminished ability to tolerate halo immobilization and reduced potential for osseous union. The aim of the study was to evaluate the morbidity, mortality and outcome in elderly patients with cervical spine injuries.

\section{Materials and methods}

During the period 1994-2002, 107 elderly patients with cervical spine injuries were managed in the National Spinal Injuries Unit based in the Mater Misericordiae University Hospital, Dublin. The study included all elderly patients with cervical spine injuries presenting directly to the Emergency Department or referred from other hospitals within Ireland. The data was retrospectively collected by analysis of the national spinal unit database, hospital inpatient enquiry (HIPE) system, charts and radiological reviews. The spinal injury database includes all patients admitted with bony or ligamentous spinal injury with or without neurological deficit. Admission information obtained included age, sex, injury aetiology, types and levels of cervical spine injuries as well as neurological injury level and pattern, pre-existing medical and cervical spine abnormalities, and associated injuries. Hospitalization information obtained included surgical intervention, traction and orthosis, morbidity and mortality data, discharge plan, and initial hospitalization length of stay. Rehabilitation information was obtained from both direct examination of patients during their outpatient follow-up and by follow-up questionnaire and telephone interviewing of the patients.

Cervical spine radiology of each patient from initial workup was reviewed to verify case inclusion, characterize the spectrum of age-related finding and to classify the type of fracture. A standard cervical radiographic series consisting of anteroposterior, open mouth odontoid, and lateral radiographs was obtained in each case. If the lateral film failed to visualize the cervicothoracic junction, a swimmer's view was included. Other views or studies performed included flexion/extension views, magnetic resonance imaging, and computed tomographic scans. The stability and healing status of the fractures were determined radiologically with the help of specific criteria for osseous union, which included evidence of trabeculation across the fracture line and the absence of movement on lateral flexion-extension views. The bony injury was classified into two categories: a fracture alone or a dislocation with or without fracture. The number of contiguous and non-contiguous segments involved was also determined. Fracture of the upper cervical spine, including the levels $\mathrm{C} 0-\mathrm{C} 2$, were classified according to Harris et al. Lower cervical spine fractures, including the levels $\mathrm{C} 3-\mathrm{C} 7$, were classified on the basis of a revision of the Orthopaedic Trauma Association classification method. Among the upper cervical spine injury, Axis fractures were further classified according to Anderson and D'Alonso [1] method. These elderly patients were subdivided into young elderly (6575 years) and old elderly ( $>75$ years). On the basis of mechanism of injury, they were separated into two groups. Low-energy injuries (falls from standing or seated height), and high-energy injuries (falls from greater than standing height, motor vehicles accidents, pedestrians knocked down by a car or by an animal). Outcome was assessed using the cervical spine outcome questionnaire from Johns Hopkins School of Medicine. This outcome questionnaire form is a comprehensive, verified outcome assessment for cervical spine injury, which contains six composite measures (neck pain, shoulder pain, functional disability, physical symptoms, health care utilization and psychological distress). Scores for each measure were rescaled in the range $0-100$. Questionnaires were sent by post to all patients identified. Non-responders were subsequently contacted by phone, if possible, to complete the questionnaire.

\section{Results}

Among the 107 patients identified, 8.4\% were direct admissions from our Accident and Emergency department, while $91.6 \%$ were tertiary referrals from other hospitals in Ireland. There were 72 male and 35 female patients (Table 1) At the time of injury, 52\% were in the 6575 years age group (young elderly group) and $48 \%$ were over 75 years of age (old elderly group). Sixty per cent sustained low-energy injury (fall from seating or standing position), while $40 \%$ suffered from high-energy injury (motor vehicle accidents, fall from higher than standing position etc). Falls were the predominant mechanism of injury $(70 \%)$.

The level involved was atlanto-axial complex in 44 patients, sub-axial in 52 patients, whereas 11 patients had 
Table 1 Summary of clinical variables in cervical spine injury

\begin{tabular}{llll}
\hline Category & Variables & $\begin{array}{l}\text { Patients } \\
\text { no. } 107\end{array}$ & Percentage \\
\hline Age & 65-75 years & 56 & 52.3 \\
& Over 75 years & 51 & 47.6 \\
Sex & Male & 72 & 67.2 \\
Mechanism & Female & 35 & 32.7 \\
& Low energy injury & 64 & 59.8 \\
Level of Injury & High energy injury & 43 & 40.1 \\
& Atlanto-axial & 44 & 41.1 \\
& Subaxial & 52 & 48.5 \\
Neurological & No bony injury & 11 & 10.2 \\
Deficit & Incomplete & 27 & 25.2 \\
& Complete & 4 & 3.7 \\
Treatment & Intact & 76 & 71 \\
& Non-operative & 92 & 85.9 \\
& Operative & 15 & 14 \\
\hline
\end{tabular}

no bony injuries (Table 1). Ten patients developed a central cord syndrome. The pattern of injury was fracture only in $51.4 \%$ while $36.4 \%$ had vertebral dislocation with or without fracture. Forty-one per cent sustained isolated cervical spine injuries. Injuries to two adjacent vertebrae were seen in $47 \%$, of which one patient had an associated fracture in a non-contiguous cervical vertebra and two had concurrent thoracolumbar fractures. One patient received injury to three adjacent cervical vertebrae $(\mathrm{C} 4,5,6)$. Frequency and distribution of injuries to isolated as well as adjacent levels were evaluated. Isolated injury level in the upper cervical spine was dominated by $\mathrm{C} 2(26 \%)$ followed by $\mathrm{C} 6$ in lower cervical spine $(7.4 \%)$. The most common site in the cervical spine for multilevel injuries was at the C6/C7 level (13\%). However in the upper cervical spine, the $\mathrm{C} 1 / \mathrm{C} 2$ segment $(8.4 \%)$ was the most frequently involved. The other segments were less frequently affected (C2/C3 2.8\%; C3/C4 4.6\%; C4/C5 5.6\%; C5/C6 11.2\%). Injury type by vertebral level was also evaluated. In the upper cervical spine, odontoid fracture (30) was the most frequently occurring fracture, and of these, type II (22) was the most common fracture pattern. Eight patients sustained a type III odontoid fracture and there were no cases of type I fracture. In the lower cervical spinal injuries, $28 \%$ were clinically stable (isolated spinous process, laminar or anterior body compression fractures). Most of the remaining injuries were traumatic spondylolistheses. However in elderly patients, most lower cervical spine injuries are considered potentially unstable, which is supported by the fact that most of the surgically treated patients had lower cervical spine injuries. In 107 patients, 141 vertebrae were fractured or dislocated, as a result of multilevel injuries. Frequency of individual vertebral involvement was evaluated. The distribution of cervical levels showed peaks in incidence at the $\mathrm{C} 2$ and $\mathrm{C} 6 / \mathrm{C} 7$ levels. Out of the 141 cervical vertebral fractures $9.2 \%$ occurred at the atlas (C1) level, $28.3 \%$ at the axis (C2) level, $6 \%$ at $\mathrm{C} 3,11 \%$ at $\mathrm{C} 4$, $13 \%$ at $\mathrm{C} 5,22 \%$ at $\mathrm{C} 6$ and $14 \%$ at $\mathrm{C} 7$. At the time of initial neurological examination, $25 \%$ patients had incomplete neurological deficit, 3\% had complete neurology and 72\% were neurologically intact. Associated injuries were reported in around $24 \%$ of the patients (Table 3), and included the following: long bone fractures $13 \%$; chest injuries $3.7 \%$; brain injury $2.8 \%$; facial injury $1.8 \%$; brachial plexus injury $1.8 \%$.

\section{Cervical immobilization}

External immobilization was used in $86 \%$ of patients in our study, while $14 \%$ underwent surgical stabilization (Table 1). Among the non-operative group, four fractures had delayed union (9-11 months), one of them an odontoid type III fracture and the remainder odontoid type II fractures. Three fractures failed to unite; all were odontoid type II fractures. None of these underwent surgical stabilization. One patient was unfit for anaesthesia due to medical co-morbidities, another patient refused surgery, and a third had lateral flexion/extension radiographs demonstrating stable non-union. The mean in-hospital stay was 10 days (2-90 days) for patients treated with external immobilization (halo, Minerva, Miami-J), with an average healing time of 3-4 months. Internal immobilization was carried out in 15 patients (14\%). Anterior stabilization was undertaken in three patients, two primarily (central cord syndrome and $\mathrm{C} 4$ fracture) and one secondarily, for radiological instability after treatment of $\mathrm{C} 6 / \mathrm{C} 7$ subluxation in a cervical orthosis. Twelve patients underwent primary posterior stabilization. Ten patients had lower cervical spine subluxation or instability, and two patients had upper cervical spine fracture and subluxation. All patients in this group achieved osseous union and there was no evidence of late spinal instability in these patients.

\section{Morbidity}

18.6\% developed complications during follow-up. Two patients developed pressure ulceration in Minerva casts, one in the occipital area and the other on the chin. These patients were treated in Miami-J collars and the ulcers healed with local measures only. Three patients treated in a halo had problems with pin site infections, ultimately requiring halo removal and further treatment in a Minerva cast. The infected pin sites were treated with local measures and antibiotics. MRSA was isolated from the wounds 
of all three patients. Skin erythema was observed in one patient treated in a hard collar, and this was subsequently replaced by a soft collar. Fracture position was lost in three patients treated in a halo. These were readjusted initially, but ultimately all three halos were removed and these patients were treated in a hard collar and two Minerva jackets. Minerva jackets loosened in three patients. Two were reapplied and the third patient underwent atlantoaxial arthrodesis by anterior plating. One patient developed aspiration pneumonia. Another patient developed lower respiratory chest infection in the perioperative period, requiring transfer to the intensive care unit. One patient suffered from recurrent gastrointestinal bleeding. Transient swallowing difficulty was noted in one patient after anterior cervical spine stabilization.

\section{Mortality}

In our study, in-hospital mortality was $11.2 \%$, while $19 \%$ died post-discharge during follow-up, yielding an overall mortality of $30 \%$ in elderly patients with cervical spine fractures. Among the in-hospital group eight patients died during their initial hospitalization (2-12 days) either from the complications of polytrauma or due to pre-existing medical conditions and four patients died at over 4 weeks from their injury. The majority were managed conservatively (9-orthosis, 2-halo brace) apart from one patient who died post-operatively ( $\mathrm{C} 3 / \mathrm{C} 4$ spinal fusion). Among the inpatient mortalities at over 4 weeks, two patients both with odontoid type II fractures developed lower respiratory tract infections requiring intensive care unit beds but failed to respond to treatment and died. A third patient, with fracture subluxation at $\mathrm{C} 4 / \mathrm{C} 5$ and complete neurological deficit below C5, suffered a cardiac arrest and subsequently died from multiple organ dysfunction syndrome. The fourth patient with $\mathrm{C} 3 / \mathrm{C} 4$ subluxation died of a cardiac arrest after spinal fusion.

In the early mortality group, all patients were treated non-operatively; two were immobilized in halo braces and the remainder in orthoses (Miami-J). One patient with C6/ C7 fracture subluxation and head injury died of a pontine haemorrhage after 6 days. Another patient with C6/C7 subluxation, died of cardiopulmonary complications of polytrauma within 12 days. Of patients with upper cervical spine injuries, one with $\mathrm{C} 1$ lamina and facet fracture died of myocardial infarction on the second day and one patient with odontoid type II fracture died of respiratory arrest. The remaining four patients, all with lower cervical spine injuries with incomplete neurological deficits, died of cardiopulmonary problems. All of the 15 mortalities in the follow-up group died of pre-existing medical conditions.

\section{Outcome}

We analysed the outcome of cervical spine injuries using the Cervical Spine Outcome Questionnaire described by BenDebba and Heller [6]. This questionnaire has been shown to have high reliability and responses to change after treatment. Low score signifies better outcome.

Sixty-seven patients $(70 \%)$ completed the form, 20 patients (19\%) were deceased at review and 8 patients $(7 \%)$ were uncontactable. Functional disability was more marked in the patients with neurologically deficit at time of injury. Outcome of the injury was related to increasing age, co-morbidity and the severity of neurological deficit.

\section{Discussion}

Cervical spine injuries are not uncommon in the elderly population, and are more frequently the result of a relatively minor trauma. Upper cervical injuries are the most common, have the longest hospital treatment, and are caused mainly by low energy falls [7]. At present, data from different parts of the world report a decreasing neck injury incidence for age groups under 65 years, while the incidence is constant or increasing for elderly. The incidence of cervical spine injuries in the elderly is greater than that among all other age groups. This distribution has been attributed to the tendency for the elderly to sustain simple falls, to have a greater risk of motor vehicle accidents, and to the biochemical attrition associated with senile osteopenia. Our study support previous studies, which reported that a simple fall was the most common mechanism of injury among the elderly, particularly females [10, 11, 13, 18]. Patients sustaining low-energy falls (standing or seated heights) are more likely to injure their upper cervical spine in contrast to patients who are involved in high-energy injuries (falls from greater than standing height, motor vehicle accident [10]. Previous studies suggest that old elderly patients ( $>75$ years), compared with young elderly patients (65-75 years), are more likely to sustain cervico-cranial injuries, irrespective of the mechanism [10, 18]. Our results supported those studies. In our study, the predominant mechanism of injury was fall in $70 \%$ of patients, and motor vehicle accident in the other $30 \%$. The elderly population may be subdivided into young elderly (65-75 years) and old elderly ( $>75$ years) [14]. Comparing the mechanism of trauma in our age-based subpopulations, we noticed that almost equal proportion of patients $(>60 \%)$ in each subgroup sustained cervical spine injuries during falls from standing or seated height and the remainder $(<40 \%)$ were injured in motor vehicle accidents. These results are in contrast with other series $[10,18]$. 
These finding suggests that apparently low-energy injury mechanism in the elderly population should be considered high risk for cervical spine injury and should be investigated appropriately.

Degenerative osseous changes are known to influence the site of cervical spine injuries. Normally, in a younger individual, C4-C7 are the most mobile segments of the cervical spine. With degenerative changes, these same segments become stiffer and the $\mathrm{C} 1-\mathrm{C} 2$ segment becomes the most mobile portion of the cervical spine in elderly patients [7, 10, 11], predisposing the atlanto-axial segment to injury with relatively trivial trauma.

Injury distribution in our study supports this stiffening theory, with the $\mathrm{C} 2$ vertebra as the most frequently injured level followed by $\mathrm{C} 1$ level in the upper cervical spine. Odontoid peg fractures in the elderly are the most common and serious injury. This can be a significant source of morbidity and mortality in all age groups [2, 15, 17]. It is the most frequent individual fracture of the cervical spine in person aged 65 years and older [1, 7, 10-14, 16, 18]. Odontoid fracture also occurred most frequently in isolation [13]. In our study, among the $\mathrm{C} 2$ fractures (33.6\%), odontoid fractures $(83.3 \%)$ dominated the fracture pattern. Sixty-one per cent were odontoid type II fractures, $22.2 \%$ odontoid type III fractures and no type I fractures. The remaining $16.6 \%$ were of other patterns (hangman-3, body fractures-2, lamina fracture-1). These results correlate with those of some studies [3, 14], although another had identified fractures of the atlanto-axial complex in all patients [18]. In addition to the tendency of cervical fractures in the elderly to involve the atlanto-axial complex, there is also a tendency towards combination $\mathrm{C} 1-\mathrm{C} 2$ fractures, represented by $5.6 \%$ of fractures in our study. This is consistent with the results of Sonntag and Hadley [15], who reported $2 \%$ of $\mathrm{C} 1-\mathrm{C} 2$ combination fractures from the total population of acute cervical spine injuries in their series while another study has reported the figure as much higher [18]. Our study also demonstrated a $2.8 \%$ incidence of C2-C3 combination fractures. Thus in our study, the overall incidence of atlanto-axial complex injury was $38.2 \%$ in the elderly patients. As has been reported in the previous reviews, $\mathrm{C} 2$ is the most commonly injured isolated vertebra in the elderly cervical spine. However the multilevel and combination injuries were more common in the lower cervical region in our study. As a result of multilevel injuries, 141 vertebrae in the 107 patients reviewed were fractured or dislocated. Sixty-five per cent were lower cervical vertebrae. C6 vertebra was the most frequently affected at $21.9 \%$, followed by, C7 in $14 \%$, C5 in 12\%, C4 in $10 \%$ and $\mathrm{C} 3$ in $6.3 \%$. Forty-eight per cent sustained lower cervical spine injuries and $41 \%$ injured upper cervical spine (Table 2). Thus our review demonstrated a higher proportion of lower cervical spine injuries than
Table 2 Distribution of upper and lower cervical spine injury in patients by age group, including subgroup odontoid fracture

\begin{tabular}{lll}
\hline Category & $65-75$ years & Over 75 years \\
\hline C1-C2 & 20 & 24 \\
C3-C7 & 29 & 25 \\
Odontoid fractures & 23 & 19 \\
\hline
\end{tabular}

upper cervical spine injuries in the elderly population, which is inconsistent with many other series reporting upper cervical injuries in majority of patients [7, 13, 18] Neurological injury associated with cervical trauma is less common in elderly patients, due to the higher incidence of odontoid injuries and the more minor nature of the trauma [16]. When a neurological injury does occur in the elderly, it is more commonly incomplete. In our study, 27 patients demonstrated incomplete neurological injury and 4 patients presented with complete neurological deficit. In the incomplete group, the majority (26 patients) sustained lower cervical spine injuries, whereas only one patient suffered upper cervical spine injury. Simple fall caused the injury in 19 patients, 8 patients were involved in motor vehicle accidents. Sixty-seven per cent of the patients were in the young elderly age group and $33 \%$ in the old elderly group. Among the complete neurological injuries, all patients sustained lower cervical spine injuries, all were male and all were in the young elderly age group. Neurological recovery is delayed in the elderly patients but long-term functional improvement can be expected in the neurologically incomplete patients. External immobilization (orthosis-67, halo-25) was our treatment of choice for most of the cervical spine injuries (particularly for $\mathrm{C} 1-\mathrm{C} 2$ injuries). External immobilization obviates the necessity for surgical intervention in a significant proportion of elderly patients, and the halo is the most effective external device for the atlanto-axial complex immobilization, reducing flexion, extension, and lateral bending to $4 \%$ and rotation to $1 \%$ of normal [9]. Halo apparatus can be utilized for expeditious immobilization and reduction in acute trauma, can maintain alignment during in situ surgical fusion and can allow mobilization of most elderly patients in the early post-injury period. Early mobilization is highly recommended for elderly patients, as prolonged periods of immobilization are poorly tolerated, with a high incidence of complications, mainly respiratory [8]. One study reported that, among elderly patients with odontoid fractures, one-third of those patients who underwent prolonged bed rest suffered respiratory complications, while none of the patients who mobilized early during the treatment period developed this problem. Two deaths were directly attributed to flat bed rest [8]. In our study, the stable fractures and subluxations were immobilized with an 
orthosis (Minerva cast, Miami-J collar). Unstable fractures and ligamentous injuries were stabilized with application of a halo frame in 25 patients. Most of the 15 patients treated with halo frame had atlanto-axial fractures, whereas 8 patients suffered from lower cervical spine injuries. In the lower cervical spine injuries, five patients exhibited incomplete neurological deficit. Ten out of 25 patients treated with halo braces were over 75 years of age (old elderly) and the remaining 15 patients were in the young elderly group (65-75). The majority of elderly patients appear to tolerate the halo frame relatively well. Pin sites were complicated by infection in three patients. The halo braces in these cases were replaced by Minerva casts and infection controlled with antibiotics. Halo loosening occurred in three patients, requiring readjustment and later replacement by Minerva casts. Weller reported no complications with halo immobilization [18]. There were few complications related to use of orthoses in our patients. Occipital and chin pressure ulceration were seen in two patients. Three patients complained of Minerva loosening; two were reapplied, and a third underwent internal stabilization of atlanto-axial complex with anterior plating because of persistent instability. Three patients developed non-union; all had odontoid type II fractures. When the fracture is unlikely to heal or is unstable, or if the halo device cannot be tolerated, internal fixation should be considered. Twelve patients in our series underwent posterior stabilization, whereas anterior stabilization was performed in three patients without any perioperative morbidity or instability; all have progressed to osseous union.

Outcome scores revealed significant improvement regarding neck and shoulder pain, only $15 \%$ patients reported mild pain. Abnormal physical symptoms were reported by majority of patients and almost all patients had mild to moderate functional disability. There might be some other factors contributing to these symptoms, e.g. arthritis, generalized weakness due to aging process, or medical co-morbidities. A majority of patients were still able to function independently, as their health care utilization ability had recovered significantly (Tables 3,4 ).

In summary these finding suggests that apparently lowenergy injury mechanism in the elderly population should be considered high risk for cervical spine injury and should be investigated appropriately.

Standard protocol should be used to evaluate a potential cervical spine injury $[4,5]$. Those patients, who are awake, alert, have no mental status changes, no neck pain and no neurological symptoms may be considered to have a stable cervical spine and need no radiographic imaging. All other blunt trauma patients require plain cervical spine X-ray (anteroposterior, lateral and odontoid) during their initial assessment. These and the results of physical examination
Table 3 Associated injuries

\begin{tabular}{ll}
\hline Injuries & Number of patients $(\%)$ \\
\hline Long bones fractures & 13 \\
Chest injuries & 3.7 \\
Facial injuries & 1.8 \\
Brain injuries & 2.8 \\
Brachial plexus injuries & 1.8 \\
\hline
\end{tabular}

Table 4 Outcome scores

\begin{tabular}{ll}
\hline Category & Outcome score \\
\hline Neck pain & 22.2 (range10-60) \\
Shoulder pain & 28.2 (range 10-53.3) \\
Physical symptom & 40.9 (range 20-100) \\
Functional disability & 77.0 (range 52.3-100) \\
Health care utilization & 20.0 (range 0-20) \\
Psychiatric symptom & 15.3 (range 5.5-61.1) \\
\hline
\end{tabular}

may indicate the need for further imaging (flexion/extension views, magnetic resonance or computer tomography imaging).

\section{Conclusion}

Cervical spine injuries in the elderly are clinically important, and have the potential to cause cervical spinal cord injury. Fall was the most common mode of injury and fracture of the atlanto-axial complex predominated the injury pattern. Odontoid fractures were the most frequent individual fracture in persons aged 70 years and over, and a high index of suspicion should be maintained in the elderly patient with neck pain after trauma.

Standard protocol should be used to evaluate a potential cervical spine injury $[4,5]$ as mentioned previously.

Associated neurological injury is less common due to the higher incidence of odontoid injuries and the more minor nature of the trauma. When it does occur, it is usually incomplete. Neurological recovery may be delayed in elderly patients but long-term functional improvement can be expected. External immobilization was our treatment of choice for cervical spine injuries (particularly $\mathrm{C} 1-$ C2) in the elderly. In unstable fractures that are unlikely to unite, early posterior cervical fusion or early internal fixation should be considered.

\section{References}

1. Anderson LD, D'Alonso RT (1974) Fracture of the odontoid process of the atlas. J Bone Joint Surg [Am] 56:1663-1674 
2. Akmal M, Trivedi R, Sutcliffe J (2003) Functional outcome in trauma patients with spinal injury. Spine 28(2):180-185

3. Bednar DA, Parikh J, Hummel J (1995) Management of type11 odontoid process fractures in geriatric patient: a prospective study of sequential cohorts with attention to survivorship. J Spinal Disord 8:166-169

4. Brohi K, Macdonald JW (2000) Evaluation of unstable cervical spine injury; a 6-year experience. J Trauma 49:76-80

5. Banit DM, Grau G, Fisher JR (2000) Evaluvation of the acute cervical spine: a management algorithm. J Trauma 49:450-456

6. BenDebba M, Heller J (2002) Cervical spine outcomes questionnaire; its development and psychometric properties. Spine 27/ 19:2116-2123

7. Brolin K (2003) Neck injuries among the elderly in Sweden. Inj Control Saf Promot 10(3):155-164

8. Hanigan WC, Powel FC, Elwood PW, Henderson JP (1977) Odontoid fractures in cervical orthosis; a study comparing their effectiveness in restricting cervical motion in normal subjects. J Bone Joint Surg (Am) 59A:332-339

9. Johnson RM, Hart DL, Simmons EF, Ramsby GR, Southwick WO (1977) Cervical orthosis; a study comparing their effectiveness in restricting cervical motion in normal subjects. J Bone Joint Surg (Am) 59A:332-339
10. Lomoschitz FM, Blackmore CC, Mirza SK, Mann FA (2002) Cervical spine injuries in patients 65 years old and older. AJR 178:573-577

11. Olerud C, Andersson S, Svensson B, Bring J (1999) Cervical spine fractures in the elderly, factors influencing survival in 65 cases. Acta Orthop Scand 70(5):509-513

12. Pepin JW, Bourne RB, Hawkins RJ (1985) Odontoid fractures, with special reference to the elderly patient. Clin Orthop 193:178-183

13. Ryan MD, Henderson JJ (1992) The epidemiology of fractures and fracture-dislocation of the cervical spine. Injury 23(1):38-40

14. Ryan MD, Taylor TK (1993) Odontoid fracture in the elderly. J Spinal Disord 6(5):379-401

15. Sonntag VKH, Hadley MN (1991) Management of upper cervical spinal instability. Neurosurg Update 11:222-233

16. Spivak JM, Weiss MA, Cotler JM (1994) Cervical spine injuries in patients 65 and older. Spine 19:2302-2306

17. Seybold EA, Bayley JC (1998) Functional outcome of surgery and conservatively managed dens fracture. Spine 27(17):18371845

18. Weller SJ, Malek AM, Rossitch E (1997) Cervical spine fracture in the elderly. Surg Neurol 47:274-281 